\title{
Púrpura trombocitopénico trombótico con respuesta exitosa a vincristina
}

\author{
Francisco Espinoza ${ }^{1 a}$, José Luis Leal ${ }^{1 a}$, G uillermo Arenas ${ }^{2}$. \\ Successful treatment of thrombotic \\ thrombocytopenic purpura with \\ vincristine report of one case
}

Thrombotic thrombocytopenic purpura presents as a multisystemic disease with thrombocytopenia, microangiopathic bemolytic anemia, fever, neurological and renal involvement. We report a 24 years-old male presenting with purpura and a generalized seizure. His blood tests showed an hemolytic anemia, unconjungated byperbilirubinemia, increased lactated dehydrogenase, thrombocytopenia and impairment of renal function. He was initially treated with daily plasmapheresis and steroids without improvement. Due to persistence of the disease, he was treated with two doses of intravenous vincristine in four days, with clinical and laboratory improvement. He was discharged 40 days after the last dose of vincristine, in good conditions (Rev Méd Chile 2007; 135: 1572-6).

(Key words: Purpura, thrombocytopenic; Purpura, thrombotic thrombocytopenic; vincristine)

Recibido el 21 de noviembre, 2006. Aceptado el 10 de abril, 2007.

${ }^{1}$ Facultad de Medicina, Universidad de Los Andes. ${ }^{2}$ Unidad de Cuidados Intensivos, Servicio de Medicina Interna, Hospital Parroquial de San Bernardo. Santiago de Chile.

anterno de Medicina.

$\mathrm{E}_{\mathrm{f}}^{1}$ 1 púrpura trombocitopénico trombótico (PTT) forma parte del grupo de las microangiopatías trombóticas. En él se produce una agregación plaquetaria microvascular con formación de microtrombos plaquetarios sin fibrina. Se caracteriza por trombocitopenia, anemia hemolítica microangiopática, fiebre, compromiso neurológico y renal. Tiene una mortalidad cercana a 20\%.

Presentamos un caso de PTT, sin respuesta al tratamiento clásico, en el que se logra una rápida mejoría con vincristina.

$\overline{\text { Correspondencia a: José Luis Leal Fernández. Las Vertien- }}$ tes \# 1159, Las Condes, Santiago de Chile. Tel. 2431474 / 09-98860485. E mail: jlleal@gmail.com

\section{Caso Clínico}

Hombre de 24 años, previamente sano. Consultó por cuadro de 7 días de evolución de dolor cólico en fosa lumbar izquierda, asociado a hematuria moderada. Se diagnosticó un cólico renal, indicándose tratamiento ambulatorio. En los siguientes cuatro días tuvo una evolución tórpida, agregándose compromiso del estado general y disnea severa. Al examen físico se encontró febril, ictérico y con lesiones purpúricas en brazos.

El cuadro clínico empeoró en las primeras horas de hospitalización, con generalización del púrpura y compromiso de conciencia que culminó en una convulsión tónico-clónica, sin focalización neurológica ni signos meníngeos. Se decidió ingreso a la Unidad de Cuidados Intensivos (UCI), 
Tabla 1. Exámenes microbiológicos e inmunológicos

\begin{tabular}{|lll|}
\hline & Microbiología & Inmunología \\
\hline Cultivos: & Hemocultivo & Coombs directo e indirecto \\
& Urocultivo & Factor Reumatoídeo \\
& Coprocultivo & Complemento C3 - C4 \\
& & ANA - AntiDNA \\
Serología: & VIH, Hepatitis A, B, C, & Anticardiolipinas \\
& VDRL, Leptospira, T. Cruzi, & ANCA \\
Mycoplasma. & Antimembrana basal \\
\hline
\end{tabular}

conectándosele a ventilación mecánica (VM). Se tomaron múltiples muestras para cultivo bacteriano e inició antibioticoterapia de amplio espectro.

En el hemograma destacó un recuento eritrocitario (GR) de $2,5910^{6} / \mathrm{mm}^{3}$, hemoglobina ( $\mathrm{Hb}$ ) de $7,3 \mathrm{~g} / \mathrm{dL}$, hematocrito (Hto) de $21,4 \%$ y recuento plaquetario de $10 \times 10^{3} / \mathrm{mm}^{3}$, índice corregido reticulocitario de $2 \%$, con esquitocitos en el frotis. Bilirrubinemia indirecta de $3,56 \mathrm{mg} / \mathrm{dL}$, LDH de $2.477 \mathrm{U} / \mathrm{L}$, creatinina $(\mathrm{Cr})$ de $1,8 \mathrm{mg} / \mathrm{dL}$ y nitrógeno ureico (NU) de $27,8 \mathrm{mg} / \mathrm{dL}$. Sedimento urinario activo con hematuria (30 eritrocitos por campo) y proteinuria de $300 \mathrm{mg} / \mathrm{dL}$ en muestra aislada.

Las pruebas de coagulación, los estudios inmunológico y microbiológico resultaron negativos (Tabla 1). El mielograma mostró hiperplasia eritroblástica marcada. La radiografía de tórax, la ecografía abdominal y la tomografía axial computarizada (TAC) cerebral no mostraron hallazgos de relevancia. No se midió actividad de ADAMTS 13. Al segundo día de hospitalización, con el diagnóstico de PTT, se iniciaron plasmaféresis, con un recambio promedio de 1,5 volemias diarias (30 $\mathrm{ml} / \mathrm{kg}$ ). Al sexto día el paciente persistía febril, anémico, con Hto de 24,3\% y $\mathrm{Hb}$ de $8,1 \mathrm{mg} / \mathrm{dL}$, recuento plaquetario de $7 \times 10^{3} / \mathrm{mm}^{3}$, LDH de $2.600 \mathrm{U} / \mathrm{L}$, bilirrubina indirecta de $2,5 \mathrm{mg} / \mathrm{dL}, \mathrm{Cr}$ de $1,4 \mathrm{mg} / \mathrm{dL}$ y $\mathrm{NU}$ de $25,5 \mathrm{mg} / \mathrm{dL}$. Se agregó metilprednisolona en dosis inumunosupresora $(1,5 \mathrm{mg} / \mathrm{kg})$. A los catorce días, luego de una nueva convulsión y sin evidencias de mejoría clínica ni de laboratorio (Tabla 2), se agregó vincristina, en dosis de $1 \mathrm{mg}$ iv, repitiéndose igual dosis cuatro días más tarde (día 18).

Evolucionó con recuperación de los parámetros hematológicos y de la función renal, retirándose de VM (Tabla 2, Figuras 1 y 2). Desaparecieron la ictericia y las lesiones purpúricas, y no volvió a presentar convulsiones. Al día 18 de hospitalización, se suspendieron las plasmaféresis y corticoides.

Al $26^{\text {to }}$ día de hospitalizado, asintomático y con valores de laboratorio prácticamente normales, se trasladó a intermedio. A los 40 días se otorgó el alta. A los 18 meses de seguimiento no han habido signos de recidiva de enfermedad.

Tabla 2. Evolutividad de los parámetros de laboratorio más importantes

\begin{tabular}{|lcccccccc|}
\hline Día de hospitalización & $1^{\circ}$ & $6^{\circ}$ & $10^{\circ}$ & $13^{\circ}$ & $16^{\circ}$ & $20^{\circ}$ & $26^{\circ}$ & $40^{\circ}$ \\
\hline Hematocrito (\%) & 21,4 & 24,3 & 24,1 & 20 & 25,7 & 28 & 30,8 & 38,2 \\
Hemoglobina (mg/dl) & 7,3 & 8,1 & 8,1 & 8,9 & 9,1 & 9,9 & 13,9 & 14,1 \\
Plaquetas (mm $\left.{ }^{3}\right)$ & 10000 & 7000 & 9000 & 13000 & 41000 & 65000 & 167.000 & 313.000 \\
LDH (U/L) & 2477 & 2600 & 2778 & 6430 & 4322 & 2100 & 720 & 415 \\
Bili indirecta (mg/dl) & 3,56 & 2,5 & 4,5 & 5,31 & 3,11 & 2,5 & 0.66 & 0,57 \\
Creatinina/Bun (mg/dl) & $1,8 / 27,8$ & $1,4 / 25,5$ & $1,45 / 29,3$ & $1,4 / 36$ & $1,4 / 40,6$ & $1,3 / 35,8$ & $0,9 / 17$ & $0,9 / 16,7$ \\
\hline
\end{tabular}

Se ensombrecen aquellos días posteriores al uso de la Vincristina (días 14 y 18) 
Figura 1. Evolutividad del recuento plaquetario y valores de LDH.

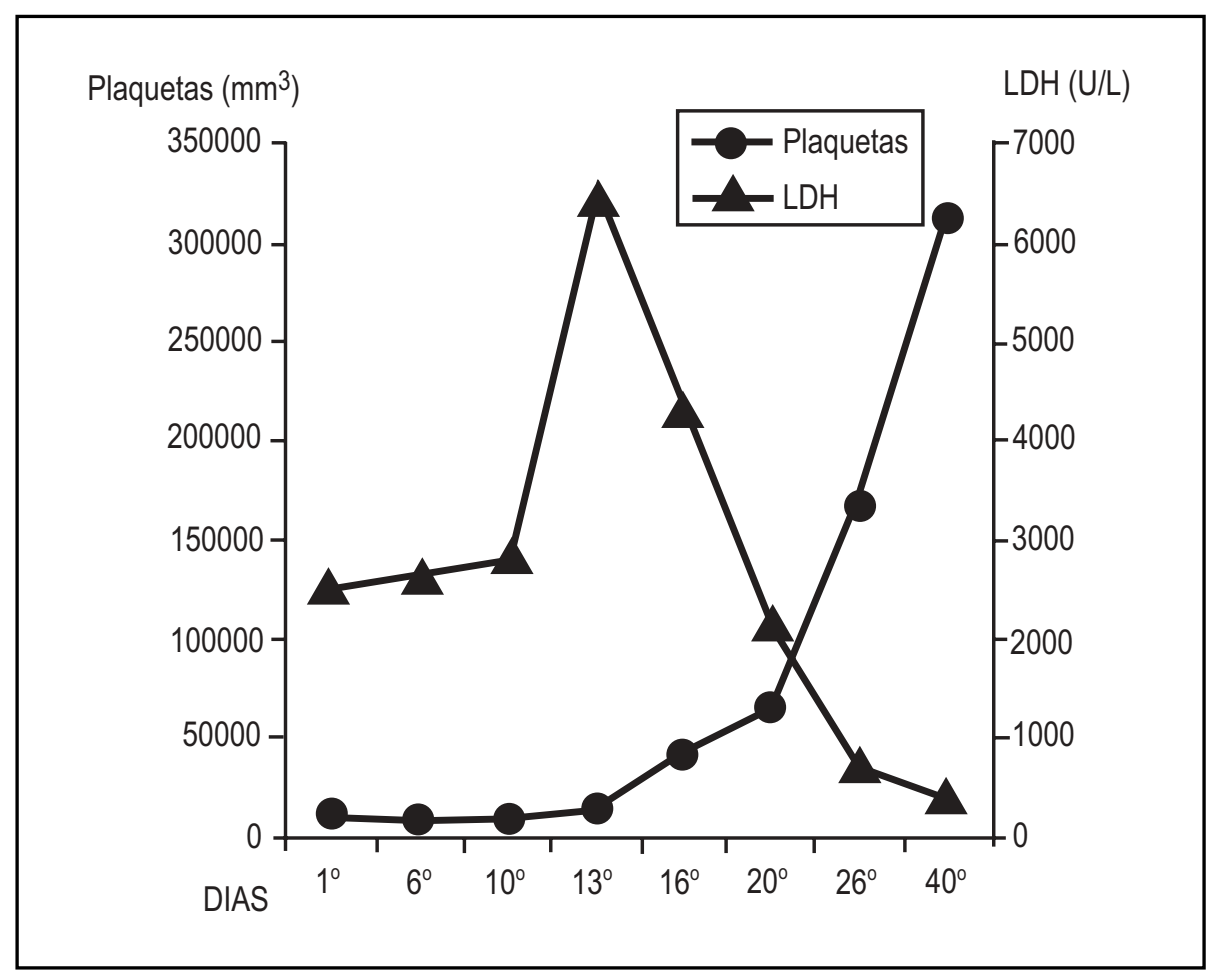

La utilidad de la vincristina se ve reflejada en el quiebre de los valores del recuento plaquetario y de LDH alrededor de los días 15 y 16. Esta mejoría es mantenida normalizándose ambos parámetros alrededor del día 40.

DISCUSIÓN

Descrito por primera vez por Moschcowitz en 1924 ${ }^{1}$, el PTT es un síndrome de carácter severo y multisistémico, donde se produce una agregación plaquetaria microvascular con microtrombos plaquetarios sin fibrina, generando trombocitopenia por consumo, isquemia tisular por oclusión arterial y anemia hemolítica por fragmentación mecánica de los eritrocitos. Afecta fundamentalmente al sistema nervioso central (SNC), gastrointestinal y, en menor intensidad, al glomérulo renal ${ }^{2,3}$.

En la década de 1980-89, se dieron los primeros pasos hacia el entendimiento etiopatogénico del PTT, descubriéndose los multímeros del factor von Willebrand (FvW) "inusualmente largos". Normalmente, las células endoteliales sintetizan y liberan FvW, que en la superficie endotelial es escindido en multímeros más pequeños mediante la acción de una metaloproteasa clivadora, denominada ADAMTS 13. Cuando existe una disminución en su actividad, ya sea por su ausencia, mutaciones en su estructura, autoanticuerpos o déficit de unión con el endotelio, la ADAMTS 13 no puede actuar sobre el FvW, liberándose a la circulación grandes multímeros que ocasionan agregación plaquetaria masiva sin fibrina, adhesión leucocitaria, consumo del complemento, flujo turbulento, fragmentación eritrocitraria e isquemia tisular ${ }^{2,3}$.

Hoy se reconocen PTT con actividad de ADAMTS 13 ausente y disminuida. Los primeros, definidos por actividad de metaloproteasa menor a 5\%, corresponden a PTT congénitos o por autoanticuerpos contra la enzima. Aquellos con actividad disminuida, mayor a 5\%, corresponden a un conjunto de entidades, tales como infecciones (VIH, mycoplasma pneumoniae), neoplasias (leucemia mieloide aguda, carcinoma pulmonar, pan- 
creático, prostático) y quimioterápicos, en los que no se ha podido establecer una relación causal con los factores descritos en su patogenia ${ }^{4,5}$.

No determinamos el nivel de ADAMTS 13. No obstante, se descartó la presencia de infecciones, neoplasias y drogas relacionadas.

El diagnóstico se basa en la pentada compuesta por fiebre, compromiso del SNC, púrpura trombocitopénico, anemia hemolítica microangiopática y compromiso renal, evidente hasta en 80\% de los pacientes. Éste se manifiesta generalmente como hematuria microscópica, proteinuria no nefrótica y falla renal leve a moderada, como en nuestro caso. Al contrario, en el síndrome hemolítico urémico (SHU) el compromiso renal supera al 90\%, estando un tercio de estos pacientes anúricos al inicio del cuadro $2,3,5,8$.

Las manifestaciones neurológicas varían desde la somnolencia hasta la focalización, convulsiones y coma ${ }^{6,7}$. En nuestro caso se presentaron convulsiones y la TAC cerebral descartó hemorragias asociadas a la trombocitopenia ${ }^{7,8}$. Respecto de la anemia hemolítica, el frotis debe presentar esquistocitos y la presencia de una respuesta megacariocítica en extendidos del mielograma, lo que se presentó.

El seguimiento se realiza con la medición seriada de plaquetas y LDH, porque son los índices más sensibles en el monitoreo de la respuesta ${ }^{3,5}$. El tratamiento del PTT es el uso de plasmaféresis con plasma fresco congelado, con lo cual se logra el mejor rango de remisión y sobrevida ${ }^{10}$. La recomendación actual es iniciarlas tan pronto se sospeche el diagnóstico, a una velocidad de entre 1 a 2 volemias diarias. Se necesitarían entre 7 y 16 días para producir una remisión estable. La decisión de finalizar el tratamiento es empírica y debe estimarse según la respuesta clínica y de laboratorio, haciendo excepción de los valores de función renal, los cuales pueden permanecer alterados en forma definitiva.

En casos de refractariedad al tratamiento, puede intentarse el uso de terapias coadyuvantes ${ }^{5,10}$. Éstas son recomendadas en conjunto con la plasmaféresis, obteniéndose resultados diversos y poco concluyentes. Se han usado corticoides en dosis altas ${ }^{10}$, lo que en nuestro caso no resultó ser efectivo. Otras terapias actualmente recomendadas son rituximab, anticuerpo monoclonal antiCD20 de los linfocitos $\mathrm{B}$, entre 4 y 8 dosis semanales, que ha demostrado ser efectivo, pero de alto $\operatorname{costo}^{5,10}$. La esplenectomía se puede considerar en casos de refractariedad con plasmaféresis o recidiva ${ }^{11-13}$. Su acción estaría fundamentada en la remoción de un importante sitio de secuestro de glóbulos rojos y plaquetas, y de lesiones microvasculares oclusivas

La vincristina, por su parte, es un derivado del alcaloide de la vinca y su uso como quimioterápico está avalado en la restricción de la proliferación celular mediante la inhibición de la replicación de microtúbulos del citoesqueleto. En el PTT se considera una droga de $2^{\underline{a}}$ línea. Durante los últimos años, varios trabajos prospectivos han tenido buenos resultados con su utilización, como terapia inicial y de rescate. En éstos se usó la plasmaféresis más un esquema de vincristina en una dosis inicial que variaba entre $1 \mathrm{mg}$ al día y 1,4 mg por $\mathrm{m}^{2}$, seguido por 3 dosis de $1 \mathrm{mg}$ cada una día por medio $5,9,10,14-17$. Diferente a nuestro caso, limitado por costos. En general, luego de su aplicación, se observó mejoría clínica y del recuento plaquetario. En uno de los trabajos se registró una elevación progresiva de los títulos de ADAMTS $13^{17}$.

Se postula que la vincristina actúa mediante dos mecanismos en el PTT; restringiendo la unión entre el FvW "patológico" y las plaquetas, previniendo la agregación plaquetaria. Por otro lado, inhibiría la producción de autoanticuerpos contra el ADAMTS-13, incrementando su actividad. Éste aumento se produciría precozmente en el curso de la respuesta a vincristina y antes de lo esperable considerando la vida media de los antianticuerpos IgG. Por eso se ha postulado recientemente, que este fenómeno se debería más bien a una estimulación positiva secundaria a la unión plaquetaria con el FvW, debido a que la unión de la glicoproteína Ib $\alpha$ plaquetaria con el dominio A-1 del FvW, estimularía el clivaje de su dominio adyacente, A-2, por la ADAMTS-13 17,18. A nuestro juicio, queda por investigar la fijación plaquetaria de la vincristina, propiedad farmacodinámica inherente, y su rol en la migración de la población de linfocitos $\mathrm{B}$ y otros tipos celulares involucrados, todo esto si se considera la inhibición microtubular.

En suma, el PTT representa una enfermedad grave, con afección seria de parénquimas vitales. Es importante la sospecha precoz para el inicio 
del tratamiento clásico mencionado, él que debe estar bien prescrito, para asegurarse de identificar a pacientes refractarios. En tal caso, la vincristina

\section{REFERENCIAS}

1. Moschcowitz E. Hyaline thrombosis of the terminal arterioles and capillaries: a hitherto undescribed disease. Proc N Y Pathol Soc 1924; 24: 21.

2. Burton R, James G. Causes of thrombotic thrombocytopenic purpura-hemolytic uremic syndrome in adults. Up To Date 14.2.2006.

3. Adler S, Nast C. Thrombotic Microangiopathies. En: Greenberg A. Primer on Kidney diseases. Philadelphia: Elsevier Saunders, 2005; 265-72.

4. Mitra D, Jaffe EA, Weskier B et al. Thrombotic thrombocytopenic purpura and sporadic hemolytic-uremic syndrome plasmas induce apoptosis in restricted lineages of human microvascular endothelial cells. Blood 1997; 89:1224.

5. Moake JL. Thrombotic Microangiopathies. N EnglJ Med 2002; 347: 589-98.

6. Kelly F, Treacher D, Williams F, Hunt B, Howard R. Coma in thrombotic thrombocytopenic purpuraJ Neurol Neurosurg Psychiatry 1999; 66: 688-97.

7. Blum AS, Drislane FW. Nonconvulsive status epilepticus in thrombotic thrombocytopenic purpura. Neurology 1996: 47: 1079-81.

8. Handin RI. Trastornos de las plaquetas y de la pared vascular. En Harrison's Principles of Internal Medicine, Kasper et al. 16th Edition 2005.

9. Gutterman LA, Stevenson TD. Treatment of thrombotic thrombocytopenic purpura with vincristine. JAMA 1982; 247: 1433-6.

10. Sadier JE, Moake JL, Miyata T, George JN. Recent advances in thrombotic thrombocytopenic purpura. Hematology Am Soc Hematol Educ Program 2004: 407-23.

11. Aqui Na, Stein SH, Konkle Ba, Abrams CS, Strobl FJ. Role of splenectomy in patients with refrac- representa hoy una opción real como terapia de rescate.

tory or relapsed thrombotic thrombocytopenic purpura. J Clin Apher 2003; 18: 51-4.

12. Kappers-Kuunne MC, Wijermans P, FijnheEr R, Croockewit AJ, Van Der Holt B, De Wolf JT et al. Splenectomy for the treatment of thrombotic thrombocytopenic purpura. Br J Haematol 2005; 130: 768-76.

13. OutsChOORN UM, Ferber A. Outcomes in the treatment of thrombotic thrombocytopenic purpura with splenectomy: a retrospective cohort study. Am J Hematol 2006; 81: 895-900.

14. Ferrara F, Copia C, Annunziata M, Spasiano A, Di Grazia C, Palmieri C et al. Vincristine as salvage treatment for refractary thrombotic thrombocytopenic purpura. Ann Hematol 1999; 78: 521-3.

15. Chamouni P, Lenain P, Buchonnet G, Merle B, Bourgain C, Boyer A et al. Difficulties in the management of an incomplete form of refractory thrombotic thrombocytopenic purpura, the usefulness of vincristine. Transfus Sci 2000; 23: 101-6.

16. Ziman A, Mitri M, Klapper E, Pepkowitz SH, GOLDFINGER D. Combination Vincristine and plasma exchange as inicial therapy in patients with thrombotic thrombocytopenic purpura: one institution's experience and review of literature. Transfusion 2005; 45: 41-9.

17. Bohmm M, Betz C, Miesbach W, Krause M, Von Auer C, GeIGER H, SCHARRER I. The course of ADAMTS-13 activity and inhibitor titre in the treatment of thrombotic thrombocytopenic purpura with plasma exchange and vincristine. Br J Haematol 2005; 129: 644-52.

18. Nishio K, Anderson PJ, Zheng XL, Sadler JE. Binding of platelet glycoprotein Ibo to Von Willebrand factor domain A-1 estimulates the cleavage of the adjacent domain A-2 by ADAMTS 13. Proceedings of the National Academy of Sciences of the United States of America 2004; 101: 10578-83. 